\title{
Hepcidin regulation in Kenyan children with severe malaria and non-typhoidal Salmonella bacteremia
}

Kelvin M. Abuga,,2 John Muthii Muriuki,, Sophie M. Uyoga,, Kennedy Mwai,,3 Johnstone Makale, ${ }^{1}$ Reagan M. Mogire, ${ }^{1,4}$ Alex W. Macharia, ${ }^{1,4}$ Shebe Mohammed, ${ }^{1}$ Esther Muthumbi, ${ }^{1}$ Salim Mwarumba, ${ }^{1}$ Neema Mturi, ${ }^{1}$ Philip Bejon, ${ }^{1,5}$ J. Anthony G. Scott, ${ }^{1,6}$ Manfred Nairz, ${ }^{7}$ Thomas N. Williams ${ }^{1,5,8}$ and Sarah H. Atkinson ${ }^{1,5,9}$

${ }^{1}$ Kenya Medical Research Institute (KEMRI) Center for Geographic Medicine Research, KEMRI-Wellcome Trust Research Program, Kilifi, Kenya; ${ }^{2}$ Department of Public Health, School of Human and Health Sciences, Pwani University, Kilifi, Kenya; ${ }^{3}$ Epidemiology and Biostatistics Division, School of Public Health, University of the Witwatersrand, Johannesburg, South Africa; ${ }^{4}$ Open University, KEMRI-Wellcome Trust Research Program Accredited Research Center, Kilifi, Kenya; ${ }^{5}$ Center for Tropical Medicine and Global Health, Nuffield Department of Clinical Medicine, University of Oxford, Oxford, UK; ${ }^{6}$ Department of Infectious Disease Epidemiology, London School of Hygiene and Tropical Medicine, London, UK; ' Department of Internal Medicine II, Medical University Innsbruck, Innsbruck, Austria; ${ }^{8}$ Department of Infectious Diseases and Institute of Global Health Innovation, Imperial College, London, UK and ${ }^{9}$ Department of Pediatrics, University of Oxford, Oxford, UK

\section{Correspondence:}

Sarah H. Atkinson satkinson@kemri-wellcome.org

Kelvin M. Abuga kmokaya@kemri-wellcome.org

Received: $\quad$ May 26, 2021. Accepted: $\quad$ September 1, 2021. Prepublished: September 9, 2021. https://doi.org/10.3324/haematol.2021.279316

(2022 Ferrata Storti Foundation Haematologica material is published under a CC BY-NC license @) $(\mathbb{Q} \Theta$

\section{Abstract}

Malaria and invasive non-typhoidal Salmonella (NTS) are life-threatening infections that often co-exist in African children. The iron-regulatory hormone hepcidin is highly upregulated during malaria and controls the availability of iron, a critical nutrient for bacterial growth. We investigated the relationship between Plasmodium falciparum malaria and NTS bacteremia in all pediatric admissions aged <5 years between August 1998 and October 2019 ( $n=75,034)$. We then assayed hepcidin and measures of iron status in five groups: (1) children with concomitant severe malarial anemia (SMA) and NTS (SMA+NTS, $n=16$ ); and in matched children with (2) SMA ( $n=33)$; (3) NTS ( $n=33)$; (4) cerebral malaria (CM, $n=34)$; and (5) community-based children. SMA and severe anemia without malaria were associated with a 2-fold or more increased risk of NTS bacteremia, while other malaria phenotypes were not associated with increased NTS risk. Children with SMA had lower hepcidin/ferritin ratios (0.10; interquartile range [IQR]: 0.03-0.19) than those with CM $(0.24 ;$ IQR: $0.14-0.69 ; P=0.006)$ or asymptomatic malaria $(0.19 ;$ IQR: $0.09-0.46 ; P=0.01)$ indicating suppressed hepcidin levels. Children with SMA+NTS had lower hepcidin levels (9.3 ng/mL; IQR: 4.7-49.8) and hepcidin/ferritin ratios (0.03; IQR: 0.01-0.22) than those with NTS alone (105.8 ng/mL; IQR: 17.3-233.3; $P=0.02$ and $0.31 ;$ IQR: $0.06-0.66 ; P=0.007$, respectively). Since hepcidin degrades ferroportin on the Salmonella-containing vacuole, we hypothesize that reduced hepcidin in children with SMA might contribute to NTS growth by modulating iron availability for bacterial growth. Further studies are needed to understand how the hepcidin-ferroportin axis might mediate susceptibility to NTS in severely anemic children.

\section{Introduction}

Malaria and invasive non-typhoidal Salmonella (NTS) are major causes of illness and death among children living in sub-Saharan Africa. According to the World Health Organization (WHO), 94\% of the 409,000 malaria-associated deaths in 2019 occurred in the sub-Saharan African region, with children under 5 years of age being disproportionately vulnerable. ${ }^{1}$ In this region, NTS bacteremia is also common accounting for $80 \%$ of the estimated 535,000 global cases in 2017. ${ }^{2}$ While NTS is commonly associated with self-limiting gastroenteritis in European populations, NTS infections in African children can cause life-threatening sepsis with case fatality rates of $20-25 \% .^{2,3}$ NTS bacteremia is highly prevalent in areas with concurrent malaria ende- micity, ${ }^{4-6}$ and reduced malaria incidence has been associated with a decrease in NTS bacteremia.,8 The association between NTS and malaria has been particularly observed in children with severe malarial anemia (SMA), ${ }^{5,9-11}$ but this has not been reported in all settings. ${ }^{12,13}$

SMA may increase susceptibility to NTS bacteremia via a number of contributory pathways including sustained hemolysis, accumulation of free heme from lysed red blood cells, increased gut permeability, disruption of immune responses, and upregulation of heme oxygenase-1 (Figure 1).$^{14}$ Heme oxygenase-1 impairs neutrophil oxidative burst capacity, reduces neutrophil bactericidal activity, and promotes iron accumulation in macrophages. ${ }^{15,16}$ Recent in vitro and animal studies suggest that hepcidin, the master iron regulator, ${ }^{17}$ may also play an important role in deter- 


\section{NTS and malaria (no anemia)}

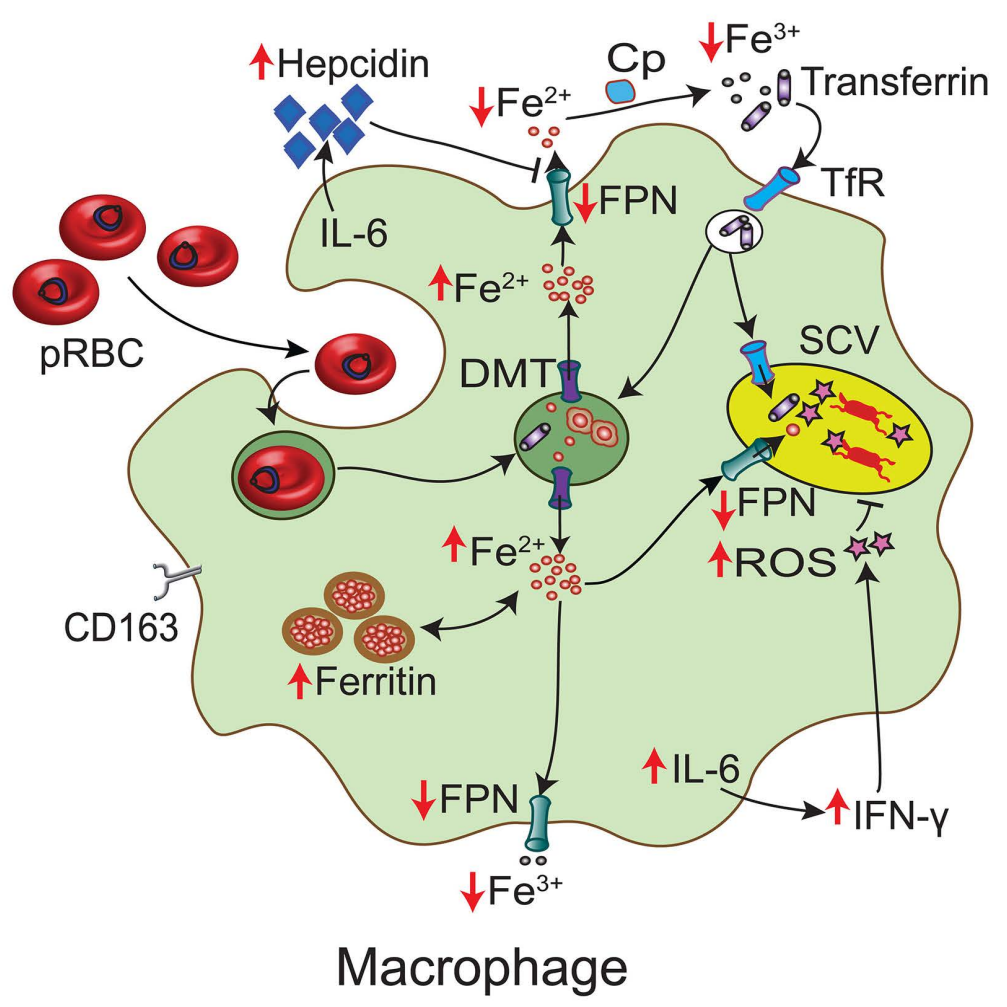

B NTS and severe malarial anemia (SMA)

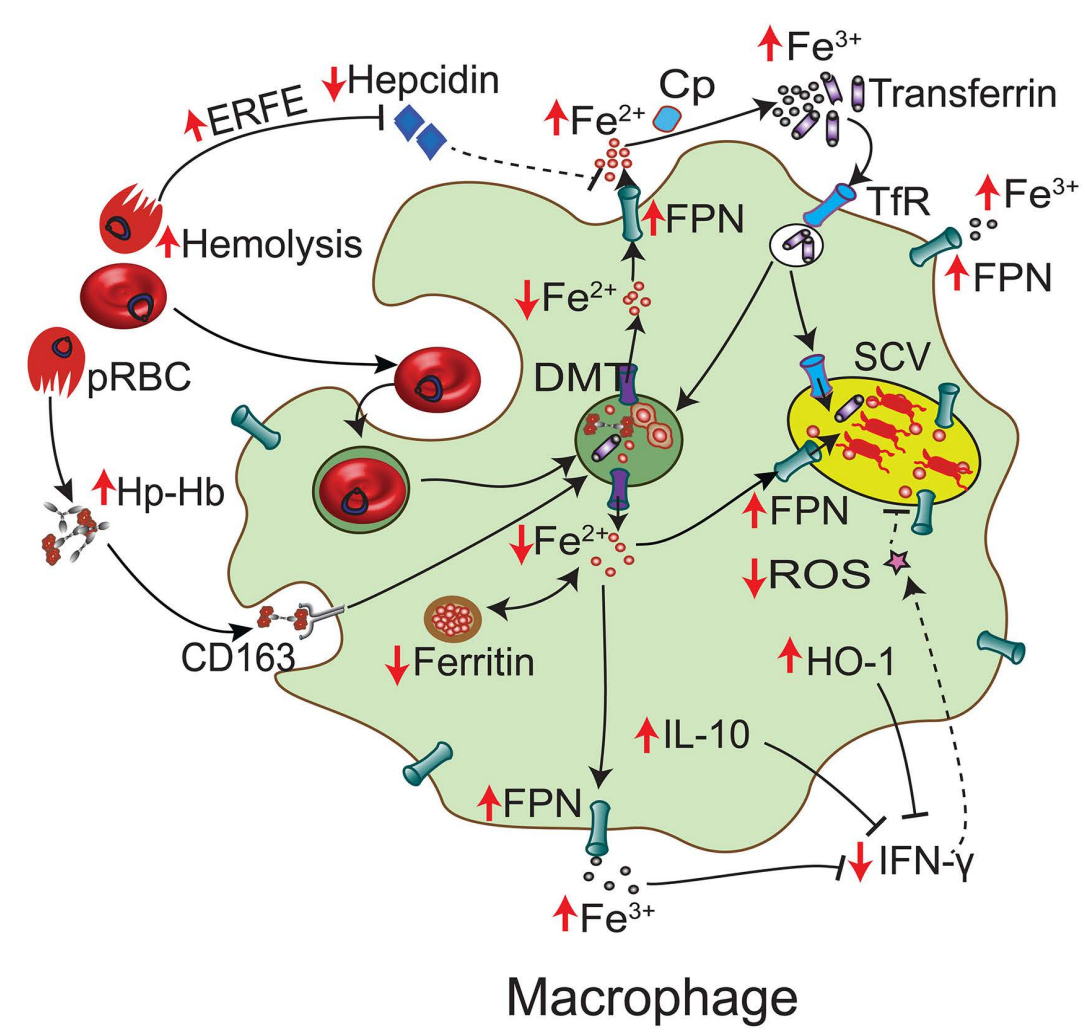

Figure 1. The hepcidin-link between severe malarial anemia and non-typhoidal Salmonella bacteremia. Low hepcidin levels in children with severe malarial anemia (SMA) may contribute to the risk of non-typhoidal Salmonella (NTS) bacteremia. (A) During malaria infection, proinflammatory responses and parasitemia induce the expression of hepcidin, the main iron regulatory hormone. Hepcidin degrades ferroportin (FPN) on the macrophage membrane and the Salmonella-containing vacuole (SCV), ${ }^{21}$ resulting in decreased iron availability for NTS bacteria. The bacteria may also utilize other iron acquisition strategies such as transferrin through transferrin receptors (TfR) in early endosomes. Proinflammatory responses, including production of interleukin (IL)-6 and interferon-gamma (IFN- $\gamma$ ), mediate killing of NTS through formation of reactive oxygen species (ROS) and other pathways. (B) In SMA, increased hemolysis and erythropoietic drive induce production of erythroferrone (ERFE), ${ }^{36}$ a hormone that downregulates hepcidin synthesis. This results in increased expression of FPN on the surface of the macrophage and the SCV. ${ }^{21}$ Heme from hemolyzed parasitized red blood cells (pRBC) and the haptoglobin-hemoglobin (Hp-Hb) complex is broken down by heme oxygenase-1 (HO-1) into equimolar amounts of iron, biliverdin and carbon monoxide. HO-1 and hemebreakdown products downregulate immune responses and promote an anti-inflammatory environment. ${ }^{15}$ The net effect of low hepcidin, increased HO-1, SMA-induced anti-inflammatory cytokines such as IL-10 and increased intra-SCV iron levels is increased proliferation of NTS bacteria. DMT-1: divalent metal transporter 1; Cp: ceruloplasmin.

mining susceptibility to NTS by controlling the availability of iron, ${ }^{18-20}$ a nutrient critical for bacterial growth and proliferation. ${ }^{16,19}$ Hepcidin degrades ferroportin, the sole iron exporter, which was recently shown to transport iron across the Salmonella-containing vacuole (SCV)..$^{21,22}$ In murine studies, low hepcidin levels and increased ferroportin expression on the SCV are associated with increased susceptibility to Salmonella Typhimurium infections (Figure 1). ${ }^{18,22}$ However, there are no studies of hepcidin in NTS infection in humans. In this study, we investigated the relationship between malaria, anemia and NTS in 75,034 hospitalized Kenyan children over a 21-year period and then estimated levels of hepcidin and other iron biomarkers in children with NTS bacteremia and malaria.

\section{Methods}

\section{Study design and participants}

Ethical approval was granted by the Scientific Ethics Review Unit of the Kenya Medical Research Institute and informed consent was provided by parents or guardians (see the On- line Supplementary Appendix). The study was conducted in Kilifi, a rural malaria-endemic area along the Kenyan coast. The estimated incidence rate of NTS bacteremia among children $<5$ years was 36.6 cases $/ 100,000$ person-years between 1998 to $2014 .{ }^{23}$ Our study included two parts:

(i) We investigated the relationship between malaria and NTS bacteremia among all pediatric admissions $(n=75,034)$ aged $\leq 60$ months admitted between August, 11998 and October, 312019 with complete age, malaria and hemoglobin data.

(ii) We then measured hepcidin, iron and inflammatory markers in five groups of children including those hospitalized with: 1) SMA and NTS coinfection (SMA+NTS); 2) SMA alone; 3) NTS alone; and 4) cerebral malaria (CM) and 5) community-based children with and without asymptomatic malaria using stored biobank samples over the 21year time period. Each child from group 1 was matched with two from each of the other hospitalized groups based on age and sex (Figure 2). The community-based children were part of an ongoing birth cohort evaluating malaria immunity, ${ }^{24}$ and their samples were selected from a single cross-sectional bleed in May 2002. 


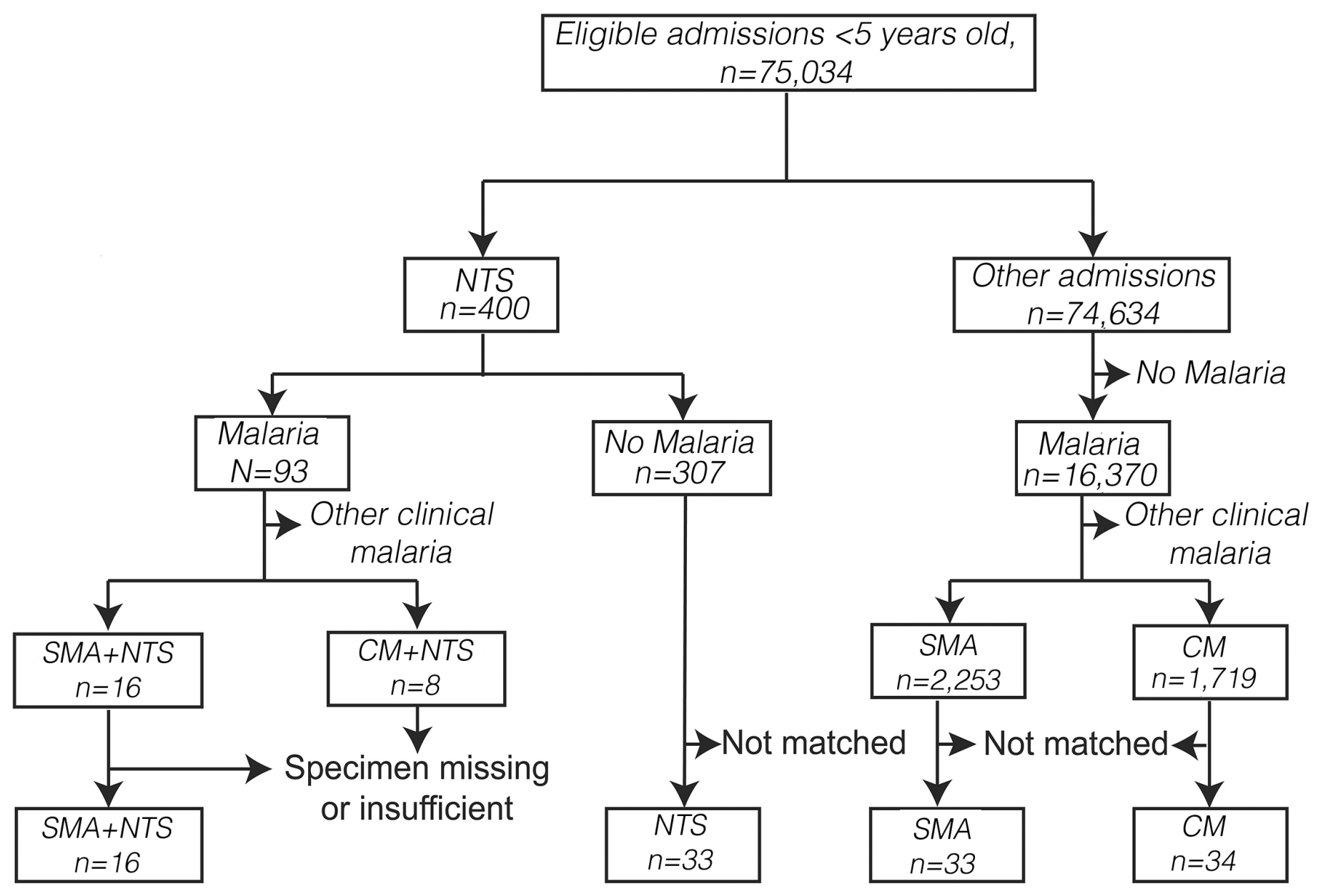

Figure 2. Selection of study participants. All children aged $\leq 60$ months with complete age and hemoglobin data admitted between August 1998 and October 2019 were included in the retrospective epidemiological analysis. Children with concomitant severe malaria and non-typhoidal Salmonella (NTS), and whose specimens were available in the Kilifi biobank, were enrolled into the iron and hepcidin sub-study. Each child was then matched with two hospitalized children with NTS alone, severe malaria anemia (SMA) alone, and cerebral malaria (CM) alone based on age and sex.

\section{Laboratory procedures}

Thick and thin blood films were stained with Giemsa and examined for Plasmodium falciparum using standard methods. Samples for bacterial culture were collected in BACTEC $^{\circledR}$ Peds Plus bottles and processed with a BACTEC9050 automated blood-culture instrument (Becton-Dickson, UK). Positive samples were sub-cultured and serological tests and biochemical test kits (API, bioMérieux) were used to confirm suspected pathogens. ${ }^{23,25}$ Bacillus species, Micrococcus species, viridans group Streptococcus, coagulase-negative Staphylococcus, and coryneforms were considered contaminants. Rapid antibody tests were used for human immunodeficiency virus 1 (HIV-1) testing. Sickle cell disease was diagnosed using polymerase chain reaction (see the Online Supplementary Appendix). Iron and inflammatory biomarkers were assayed as previously described ${ }^{26}$ (Online Supplementary Appendix).

\section{Clinical definitions}

For children with Plasmodium falciparum malaria, we defined SMA as hemoglobin $<5 \mathrm{~g} / \mathrm{dL}$ and $\mathrm{CM}$ as Blantyre coma score $<3$ according to WHO criteria. ${ }^{27}$ Severe anemia was defined as hemoglobin $<5 \mathrm{~g} / \mathrm{dL}$; fever as temperature $>37.5^{\circ} \mathrm{C}$; and NTS bacteremia as isolation of Salmonella enterica subspecies excluding Typhi or Paratyphi serovars in blood cultures.

\section{Statistical analyses}

All data were analyzed using STATA 15.1 (StatCorp. College Station, Texas, USA). For all pediatric admissions, we used univariable and multivariable logistic regression models to investigate for putative risk factors for NTS bacteremia. We used a causal directed acyclic graph to assess the suitability of covariates for multivariable analyses (Online Supplementary Figure S1), and a stepwise backward selection regression method to fit the final multivariable models (Online Supplementary Appendix). We also analyzed the relationship between SMA and risk of other bacterial organisms. In the hepcidin sub-study, continuous data were reported as medians and interquartile ranges (IQR) and compared using the Wilcoxon rank-sum test. Non-parametric Spearman's correlation evaluated associations between variables. We normalized non-normallydistributed variables by $\log _{\mathrm{e}}$-transformation and used multivariable linear regression models to adjust for inflammation (C-reactive protein) and year of admission.

\section{Results}

\section{Study of all hospital admissions}

A total of 75,034 children aged $\leq 60$ months were admitted to Kilifi County Hospital during the 21-year study 
Table 1. Factors associated with non-typhoidal Salmonella bacteremia in all hospitalized children ( $n=75,034)$.

\begin{tabular}{|c|c|c|c|c|}
\hline Characteristic & NTS, N (\%) & Hospital controls, N (\%) & OR $(95 \% \mathrm{CI})^{1}$ & $\boldsymbol{P}^{1}$ \\
\hline \multicolumn{5}{|l|}{ Clinical features } \\
\hline Age, years (IQR) & $1.07(0.55,1.90)$ & $0.98(0.18,2.18)$ & $1.01(0.93,1.08)$ & 0.87 \\
\hline Sex, male & $224 / 400(56.0)$ & 42,226/74,633 (56.6) & $0.98(0.80,1.19)$ & 0.82 \\
\hline Fever & 267/369 (72.4) & $37,886 / 61,979(61.2)$ & $1.66(1.32,2.09)$ & $<0.0001$ \\
\hline Diarrhea $^{2}$ & $126 / 400(31.5)$ & $14,187 / 74,615(19.0)$ & $1.96(1.58,2.42)$ & $<0.0001$ \\
\hline Vomiting & $127 / 388(32.7)$ & $18,061 / 73,242(24.7)$ & $1.49(1.20,1.84)$ & 0.0003 \\
\hline Severe pneumonia ${ }^{3}$ & $111 / 400(27.8)$ & $21,068 / 74,610(28.2)$ & $0.98(0.78,1.22)$ & 0.83 \\
\hline Very severe pneumonia ${ }^{4}$ & $59 / 400(14.8)$ & $7,480 / 74,606(10.0)$ & $1.55(1.18,2.05)$ & 0.002 \\
\hline Underweight ${ }^{5}$ & $204 / 340(60.0)$ & $28,479 / 68,661(41.5)$ & $2.12(1.70,2.63)$ & $<0.0001$ \\
\hline Stunting 6 & $198 / 371(53.4)$ & $27,812 / 69,510(40.0)$ & $1.72(1.40,2.11)$ & $<0.0001$ \\
\hline Wasting $^{7}$ & $180 / 380(47.4)$ & 19,493/67,493 (28.9) & $2.22(1.81,2.71)$ & $<0.0001$ \\
\hline \multicolumn{5}{|l|}{ Laboratory characteristics } \\
\hline Malaria slide positive & 93/400 (23.3) & $16,370 / 74,632(21.9)$ & $1.08(0.85,1.36)$ & 0.52 \\
\hline $\mathrm{SMA}^{8}$ & $38 / 393(9.7)$ & 2,253/74,223 (3.0) & $3.42(2.44,4.79)$ & $<0.0001$ \\
\hline Cerebral malaria ${ }^{8,9}$ & $8 / 286(2.7)$ & $1,719 / 63,244(2.7)$ & $1.00(0.50,2.02)$ & 0.99 \\
\hline Non-SMA malaria & $48 / 400(12.0)$ & 13,708/74,632 (18.4) & $0.61(0.45,0.82)$ & 0.001 \\
\hline Sickle cell disease & $14 / 400(3.7)$ & $1,115 / 74,608(1.5)$ & $2.39(1.40,4.09)$ & 0.002 \\
\hline HIV positive ${ }^{10}$ & $38 / 139$ (27.3) & $1,756 / 35,327(5.0)$ & $7.19(4.94,10.48)$ & $<0.0001$ \\
\hline $\mathrm{Hb}, \mathrm{g} / \mathrm{dL}(\mathrm{IQR})$ & $7.4(5.2,9.4)$ & $9.8(8.1,11.6)$ & $1.28(1.24,1.32)$ & $<0.0001$ \\
\hline $\mathrm{Hb}<5 \mathrm{~g} / \mathrm{dL}$ & $89 / 400(22.3)$ & $4,660 / 74,615(6.2)$ & $4.30(3.39,5.45)$ & $<0.0001$ \\
\hline $\mathrm{Hb} 5-7 \mathrm{~g} / \mathrm{dL}^{11}$ & $86 / 311(27.7)$ & 6,575/69,974 (9.4) & $3.69(2.87,4.73)$ & $<0.0001$ \\
\hline $\mathrm{Hb} 7-10 \mathrm{~g} / \mathrm{dL}^{12}$ & $147 / 225(65.3)$ & $27,867 / 63,399(44.0)$ & $2.40(1.83,3.16)$ & $<0.0001$ \\
\hline Severe anemia without malaria ${ }^{13}$ & $44 / 400(11.0)$ & $1,997 / 74,634(2.7)$ & $4.50(3.28,6.17)$ & $<0.0001$ \\
\hline
\end{tabular}

NTS: non-typhoidal Salmonella; n/N: number positive/number tested; OR: odds ratio; Cl: confidence interval; SMA: severe malaria anemia; $\mathrm{CM}$ : cerebral malaria; Hb: hemoglobin; HIV: human immunodeficiency virus. ${ }^{1} \mathrm{O} d \mathrm{~d}$ s ratios and $P$ values were derived from univariable logistic regression models; ${ }^{2}$ passage of three or more loose or liquid stools within 24 hours; ${ }^{3}$ history of cough or difficulty in breathing plus lower chest wall indrawing; ${ }^{4}$ cough or difficulty breathing plus either prostration, lethargy, hypoxia, loss of consciousness, or a history of convulsions; ${ }^{5}$ weight-for-age z-score $<-2 ;{ }^{6}$ height-for-age z-score $<-2 ;{ }^{7}$ weight-for-height z-score $<-2$ or mid-upper arm circumference $<12.5 \mathrm{~cm}$ in children $>6$ months of age using World Health Organization Child Growth Standards; $50{ }^{8}$ children with overlapping SMA and CM clinical syndromes were excluded from analysis; ${ }^{9}$ only children with Blantyre coma scale scores were included; ${ }^{10}$ data were available from February 2005 after routine HIV testing was introduced thus analyses included a limited number of children $(n=35,466)$; ${ }^{11}$ Excludes children with hemoglobin levels $<5$ $\mathrm{g} / \mathrm{dL}$; ${ }^{12}$ excludes children with hemoglobin levels $<7 \mathrm{~g} / \mathrm{dL} ;{ }^{13}$ severe anemia (hemoglobin $<5 \mathrm{~g} / \mathrm{dL}$ ) and no malaria parasites on blood film.

period and had complete data for analysis. Median age was 11.8 months (IQR: 2.2-26.1) and 42,450 (56.6\%) were male. P. falciparum malaria was identified in the blood films of 16,463 (21.9\%) hospitalized children of whom 2,291 (13.9\%) had SMA, 1,727 (10.5\%) had CM, and 416 (2.5\%) had concomitant SMA and CM. Pathogenic bacterial organisms were isolated from 3,792 (5.1\%) blood cultures. NTS bacteremia was identified in 400 (10.5\%) of the positive blood cultures. Of the NTS isolates, 309 were serotyped and $45.0 \%$ (139/309) were Salmonella enterica serovar Enteritidis and 44.3\% (137/309) were serovar Typhimurium, while $10.7 \%$ (33/309) were not typeable. The prevalence of NTS bacteremia has decreased over the years as malaria has also decreased (Online Supplementary Figure S2).

NTS bacteremia was identified in 93 of 16,463 (0.6\%) hospitalized children with $P$. falciparum malaria, including 38 of 2,291 (1.7\%) with SMA and eight of 1,727 (0.5\%) with CM. SMA was associated with a 2 -fold increased risk of NTS bacteremia in the final multivariable model (adjusted odds ratio [OR]: 2.17; 95\% confidence interval [CI]: 1.44-3.28;
$P=0.0002$; Online Supplementary Table S2). However, a positive malaria slide (OR: $1.08 ; 95 \% \mathrm{Cl}: 0.85-1.36 ; P=0.52$ ) and CM (OR: 1.00; 95\% Cl: 0.50-2.02; $P=0.99$ ) were not associated with increased risk of NTS bacteremia (Table 1). Children with malaria but without SMA had a 39\% reduced risk of NTS bacteremia (OR: 0.61; 95\% Cl: 0.45-0.82; $P=0.001$ Table 1). Children with severe anemia without malaria parasitemia also had an increased risk of NTS bacteremia in final multivariable models (adj. OR: 4.03; 95\% Cl: 2.78-5.84; $P<0.0001$; Online Supplementary Table S2). The risk of NTS bacteremia increased by $26 \%$ for each 1 $\mathrm{g} / \mathrm{dL}$ decrease in hemoglobin levels in all children (adj. OR: 1.26; $95 \% \mathrm{Cl}: 1.21-1.32 ; P<0.0001)$ and by $51 \%$ in children with malaria parasitemia (adj. OR: 1.51; 95\% Cl: 1.36-1.68; $P<0.0001)$. In final multivariable models, other risk factors for NTS bacteremia were younger age, fever, diarrhea, sickle cell disease, very severe pneumonia, underweight and, in restricted models, HIV status (Online Supplementary Table S2). SMA was not associated with increased risk of other bacterial organisms causing bacteremia (Online supplementary Figure S3). 


\section{Hepcidin sub-study}

We included 116 hospitalized children in the following groups: 1) 16 with SMA+NTS; 2) 33 with SMA alone; 3) 33 with NTS alone; and 4) 34 with CM (Figure 2); and 5) 291 community-based children with $(n=49)$ and without $(n=242)$ asymptomatic malaria parasitemia. The clinical characteristics of children in the sub-study are shown in the Online supplementary Table s3.

\section{Hepcidin levels in children with malaria}

We first compared hepcidin levels among children with malaria. Hepcidin levels were lower in children with SMA (median $31.1 \mathrm{ng} / \mathrm{mL}$, IQR: 5.5-61.2) compared to those with CM (90.7 ng/mL; IQR: 38.7-176.1; $P=0.002)$. However, both of these severe malaria groups had higher hepcidin levels than children with asymptomatic malaria parasitemia living in the community (Figure 3A). We found similar hepcidin levels in community-based children with and without asymptomatic malaria parasitemia (6.5 ng/mL; IQR: 2.0-13.1 and $3.8 \mathrm{ng} / \mathrm{mL}$;
IQR: 1.2-12.6, respectively). Hepcidin expression was suppressed in children with SMA as evidenced by a lower hepcidin/ferritin ratio (0.10; IQR: 0.03-0.19) compared to those with CM (0.24; IQR: 0.14-0.69; $P=0.006)$, or asymptomatic parasitemia (0.19; IQR: 0.09-0.46; $P=0.01$ ) (Figure 3B).

We then explored differences in putative regulators of hepcidin. Children with SMA had increased erythropoietic drive as indicated by higher soluble transferrin receptor (STfR) levels (43.3 mg/L; IQR: 30.8-65.6) than those with CM (31.2 mg/L; IQR: 23.9-45.5; $P=0.03)$, although ferritin and C-reative protein (CRP) levels did not differ between the groups (Figure 3). Hospitalized children had higher levels of ferritin, STfR, and CRP and higher P. falciparum parasite densities than those living in the community (Figure $3 \mathrm{C}-\mathrm{F}$ ).

\section{Hepcidin levels in children with malaria and non-typhoidal Salmonella}

We then considered hepcidin levels in children with malaria and NTS. Hepcidin levels were lower in children with
A
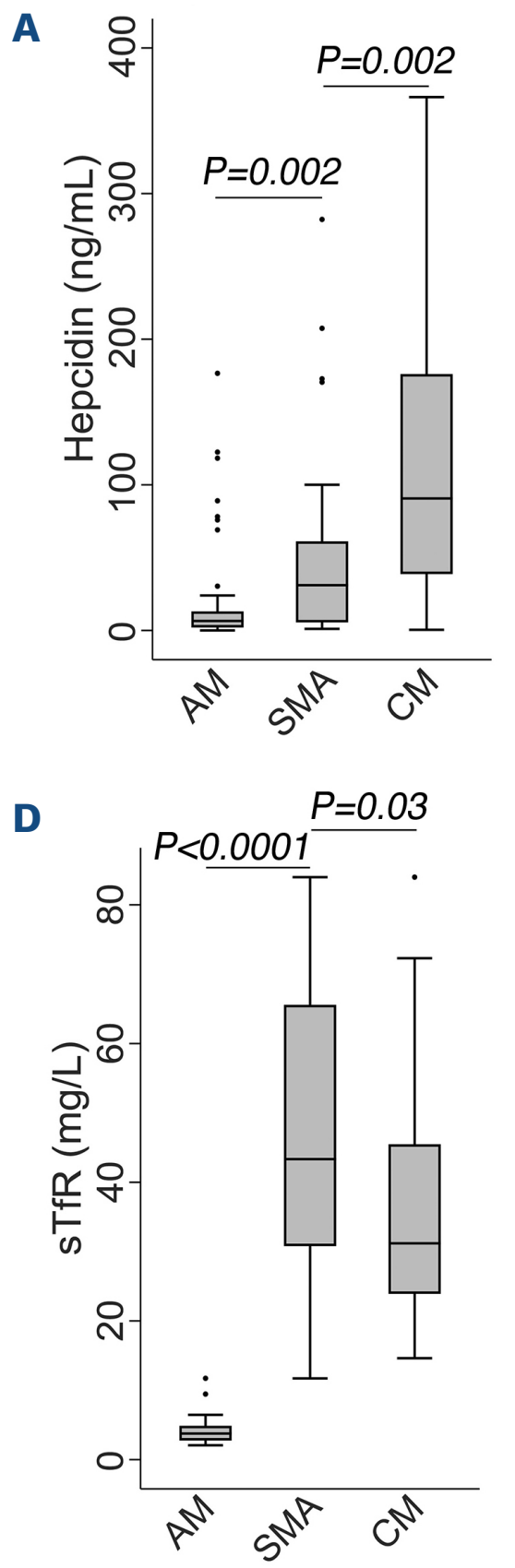

B

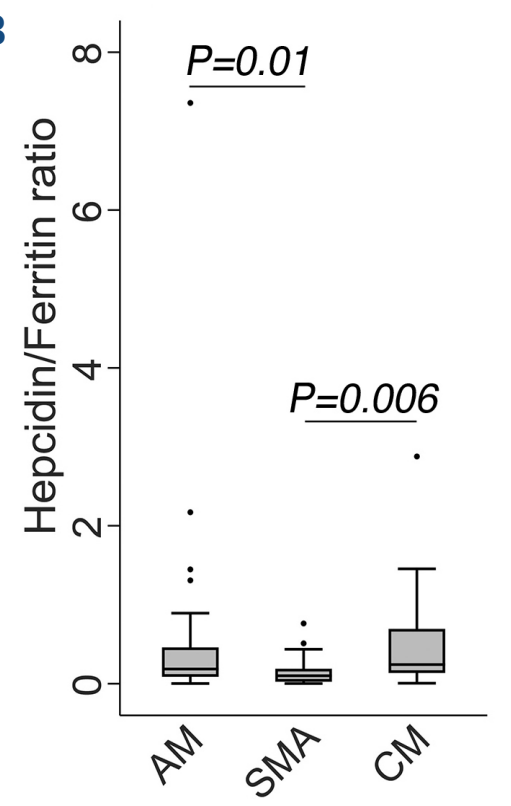

$\mathbf{E}$

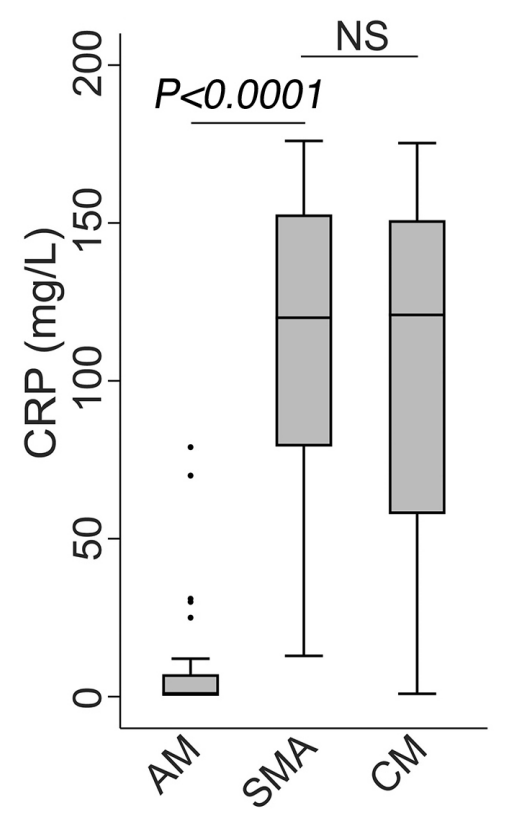

c

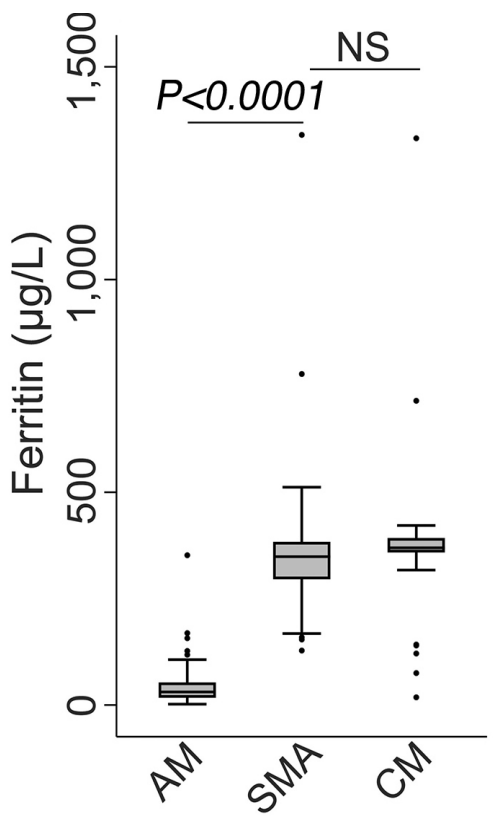

$\mathbf{F}$

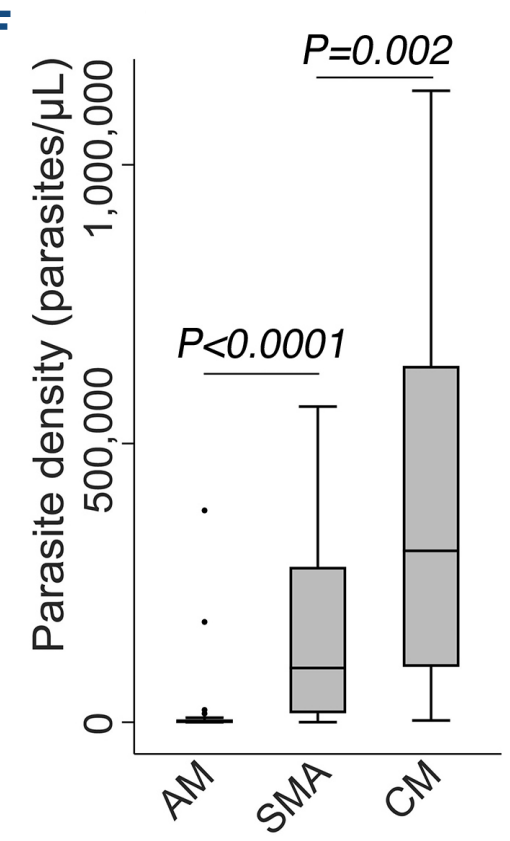

Figure 3. Iron and inflammatory biomarkers in children with malaria. Circulating levels of (A) hepcidin, (B) ferritin, (C) hepcidin/ferritin ratio, (D) soluble transferrin receptors (STfR), (E) C-reactive protein (CRP), and (F) parasite density. $P$ values from pairwise comparisons were determined by the Wilcoxon rank-sum test. NS' (not significant) indicates $P>0.05$. AM: asymptomatic malaria; CM: cerebral malaria; SMA: severe malaria anemia. 
Table 2. Hepcidin and biomarkers of iron status and inflammation in a sub-study of hospitalized and community-based children.

\begin{tabular}{|c|c|c|c|c|}
\hline Biomarker & Group & $\mathbf{N}$ & Medians (IQR) & $\boldsymbol{P}^{1}$ \\
\hline Hepcidin, ng/mL & $\begin{array}{l}\text { SMA and NTS coinfection } \\
\text { Severe malaria anemia } \\
\text { NTS bacteremia } \\
\text { Cerebral malaria } \\
\text { Asymptomatic malaria } \\
\text { Healthy controls }\end{array}$ & $\begin{array}{c}16 \\
33 \\
33 \\
34 \\
49 \\
242\end{array}$ & $\begin{array}{c}9.3(4.7,49.8) \\
31.1(5.5,61.2) \\
105.8(17.3,233.3) \\
90.7(38.7,176.1) \\
6.5(2.0,13.1) \\
3.8(1.2,12.6)\end{array}$ & $\begin{array}{c}\text { Reference } \\
0.43 \\
0.02 \\
0.004 \\
0.16 \\
0.01\end{array}$ \\
\hline Ferritin, $\mu \mathrm{g} / \mathrm{L}$ & $\begin{array}{l}\text { SMA and NTS coinfection } \\
\text { Severe malaria anemia } \\
\text { NTS bacteremia } \\
\text { Cerebral malaria } \\
\text { Asymptomatic malaria } \\
\text { Healthy controls }\end{array}$ & $\begin{array}{c}16 \\
32 \\
29 \\
28 \\
48 \\
237\end{array}$ & $\begin{array}{c}311.5(241,392) \\
348.5(296,384) \\
356.0(203,397) \\
370.0(359,393) \\
30.5(17.0,53.0) \\
16.0(8.0,26.0)\end{array}$ & $\begin{array}{c}\text { Reference } \\
\quad 0.55 \\
0.76 \\
0.23 \\
<0.0001 \\
<0.0001\end{array}$ \\
\hline Hepcidin/ferritin ratio 2 & $\begin{array}{l}\text { SMA and NTS coinfection } \\
\text { Severe malaria anemia } \\
\text { NTS bacteremia } \\
\text { Cerebral malaria } \\
\text { Asymptomatic malaria } \\
\text { Healthy controls }\end{array}$ & $\begin{array}{c}16 \\
32 \\
29 \\
28 \\
48 \\
232\end{array}$ & $\begin{array}{l}0.03(0.01,0.22) \\
0.10(0.03,0.19) \\
0.31(0.06,0.66) \\
0.24(0.14,0.69) \\
0.19(0.09,0.46) \\
0.27(0.08,0.66)\end{array}$ & $\begin{array}{c}\text { Reference } \\
0.53 \\
0.007 \\
0.01 \\
0.01 \\
0.0006\end{array}$ \\
\hline $\mathrm{sTfR}, \mathrm{mg} / \mathrm{L}$ & $\begin{array}{l}\text { SMA and NTS coinfection } \\
\text { Severe malaria anemia } \\
\text { NTS bacteremia } \\
\text { Cerebral malaria } \\
\text { Asymptomatic malaria } \\
\text { Healthy controls }\end{array}$ & $\begin{array}{c}16 \\
33 \\
32 \\
33 \\
49 \\
239\end{array}$ & $\begin{array}{c}48.1(36.8,66.9) \\
43.3(30.1,61.3) \\
38.3(30.9,67.6) \\
31.2(23.9,45.5) \\
3.8(2.7,4.9) \\
3.6(2.8,4.8)\end{array}$ & $\begin{array}{l}\text { Reference } \\
\quad 0.64 \\
0.50 \\
0.02 \\
<0.0001 \\
<0.0001\end{array}$ \\
\hline CRP, mg/L & $\begin{array}{l}\text { SMA and NTS coinfection } \\
\text { Severe malaria anemia } \\
\text { NTS bacteremia } \\
\text { Cerebral malaria } \\
\text { Asymptomatic malaria } \\
\text { Healthy controls }\end{array}$ & $\begin{array}{c}16 \\
33 \\
33 \\
33 \\
48 \\
237\end{array}$ & $\begin{array}{c}117.5(79.0,145.0) \\
120.0(79.2,152.7) \\
104.3(35.1,162.4) \\
120.9(57.8,150.9) \\
1.0(0.3,7.1) \\
0.3(0.3,2.0)\end{array}$ & $\begin{array}{l}\text { Reference } \\
\quad 0.80 \\
0.67 \\
0.93 \\
<0.0001 \\
<0.0001\end{array}$ \\
\hline
\end{tabular}

IQR: interquartile range; SMA: severe malaria anemia; NTS: non-typhoidal Salmonella; CRP: C-reactive protein; sTfR: soluble transferrin receptor; and $\mathrm{n} / \mathrm{a}$ : data not available. ${ }^{1} P$ values were derived using pairwise Wilcoxon rank sum test. ${ }^{2}$ Hepcidin/ferritin ratio was calculated by dividing hepcidin $(\mathrm{ng} / \mathrm{mL})$ by ferritin $(\mu \mathrm{g} / \mathrm{L})$.

SMA+NTS (9.3 ng/mL; IQR: 4.7-49.8) compared to those with NTS alone (105.8 ng/ml; IQR: 17.3-233.3) (Figure 4A; Table 2). Hepcidin/ferritin ratios were also lower in children with SMA+NTS (0.03; IQR: 0.01-0.22) compared to those with NTS alone (0.31; IQR: 0.06-0.66; $P=0.007$ ) (Table 2; Figure $4 \mathrm{~B}$ ). In a linear regression model controlled for CRP and year of admission, hepcidin levels were 2-fold higher in children with NTS compared to those with SMA+NTS (adj. b 1.99; 95\% Cl: 0.81-3.26; $P=0.001$ ) (Online Supplementary Table S4), although sTfR, ferritin, and CRP levels did not differ between the groups (Figure 4C-E). Only one participant in the sub-study had sickle cell disease and excluding the participant from the analysis did not alter our findings.

Hepcidin levels were positively correlated with ferritin $(r=0.38, P=0.0001)$, CRP $(r=0.31 ; P=0.0007)$, hemoglobin $(r=0.37 ; P<0.0001)$ and parasite density $(r=0.44 ; P<0.0001)$, and negatively correlated with $\operatorname{sTfR}(r=-0.37 ; P<0.0001)$ among the hospitalized children. The direction and strength of correlation between hepcidin and its predictors varied across individual groups as shown in the Online Supplementary Table S5.

\section{Discussion}

Malaria and NTS are important causes of hospitalization and death among children living in sub-Saharan Africa., ${ }^{1,2}$ In this study, we analyzed retrospective data from 75,034 hospitalized children aged $\leq 60$ months and found that SMA, but not CM or other malaria phenotypes, was associated with increased risk of NTS bacteremia. Children with severe anemia of all causes, both with or without malaria parasitemia, also had an increased risk of NTS bacteremia. In a sub-study investigating iron biomarkers, children with SMA had lower hepcidin levels than children 
A

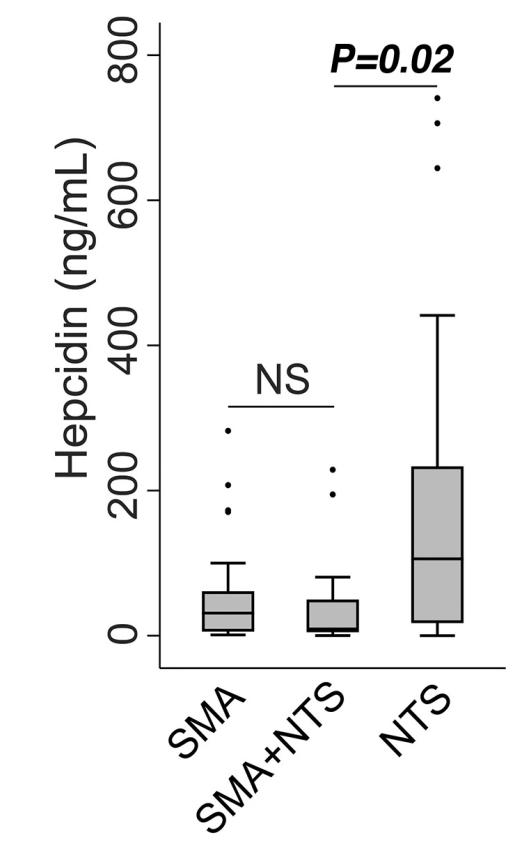

D

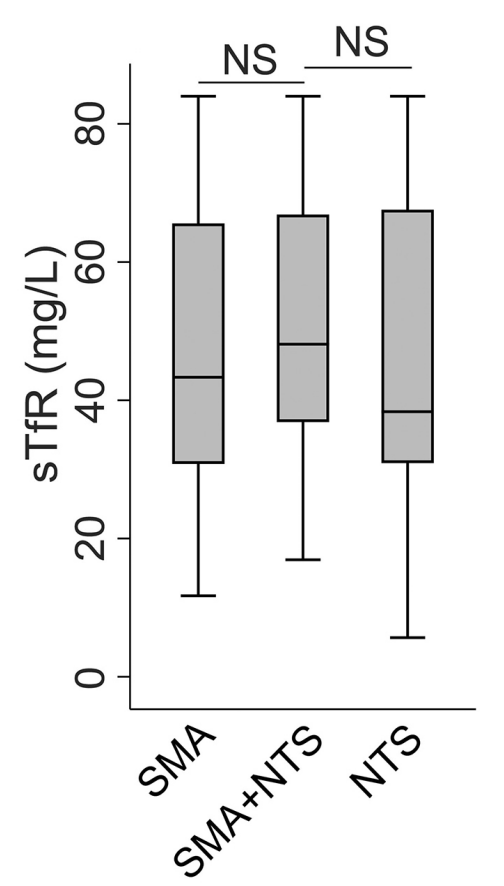

B

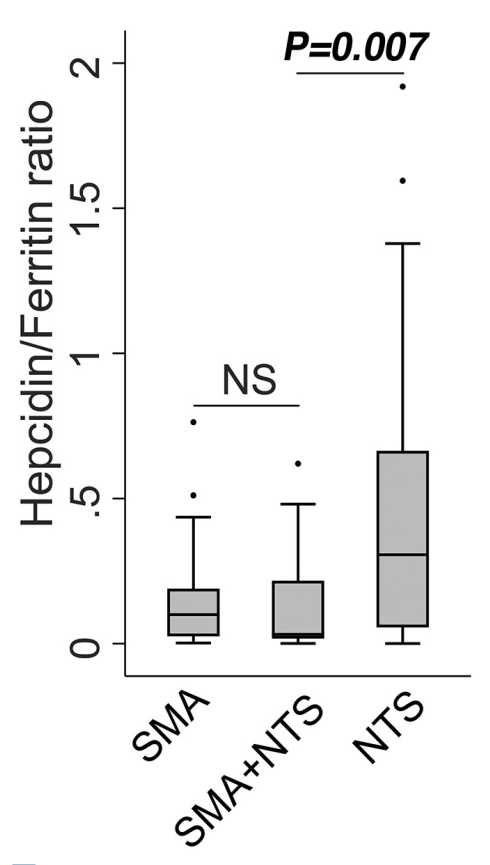

E

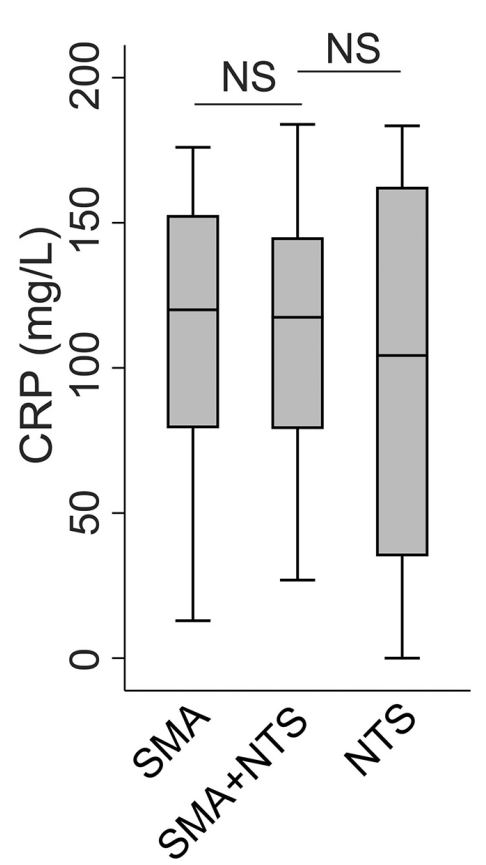

C

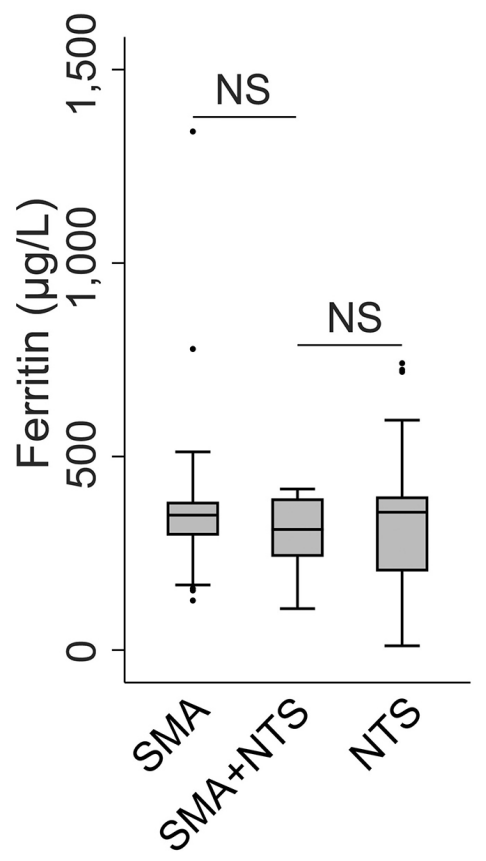

$F$

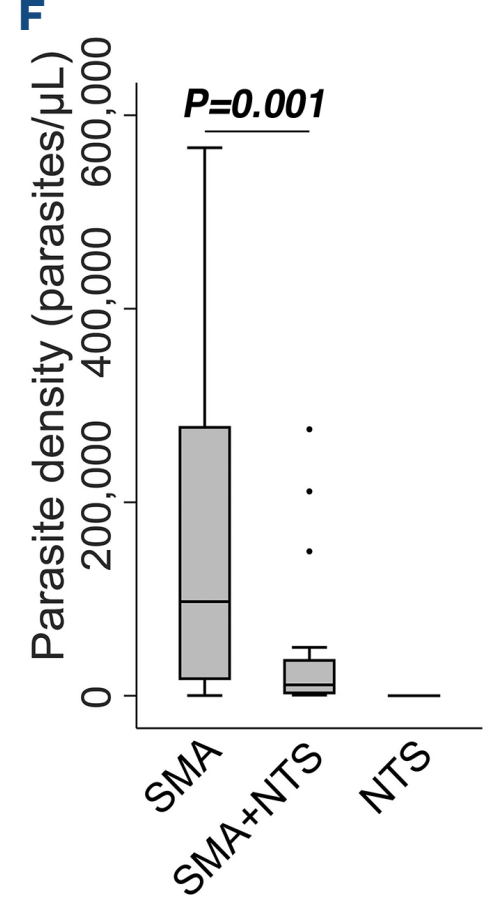

Figure 4. Iron and inflammatory biomarkers in children with severe malaria anemia and/or non-typhoidal Salmonella bacteremia. Circulating levels of (A) hepcidin, (B) ferritin, (C) hepcidin/ferritin ratio, (D) soluble transferrin receptors (sTfR), (E) Creactive protein (CRP), and (F) parasite density in children with severe malarial anemia (SMA) and/or non-typhoidal Salmonella (NTS) bacteremia. $P$ values from pairwise comparisons were determined by the Wilcoxon rank-sum test. NS' (not significant) indicates $P>0.05$.

with CM. We also found that children with SMA+NTS had lower hepcidin levels than children with NTS alone. We did not find differences in ferritin or CRP levels among hospitalized children, but children with SMA alone and SMA+NTS had lower hepcidin/ferritin ratios and higher sTfR levels. Children living in the community with asymptomatic parasitemia had lower levels of hepcidin, ferritin, CRP and STfR and lower parasite densities than hospitalized children.

Children with SMA had a 2-fold increased risk of NTS bacteremia compared to those without SMA. This risk was not observed in children with $\mathrm{CM}$ or other malaria phenotypes that excluded severe anemia. Moreover, each $1 \mathrm{~g} / \mathrm{dL}$ decrease in hemoglobin concentrations in children with malaria was associated with a $51 \%$ increase in the risk of NTS bacteremia. SMA increased the risk of NTS, but not other bacteria suggesting an NTS-specific effect rather than a generalized immunological failure to control bacteremia (Online Supplementary Figure S3). Previous studies across sub-Saharan Africa have also reported an increased risk of NTS bacteremia in children with SMA, ${ }^{5,9,10}$ but not CM..$^{10,28}$ However, these observations have not been universal. A study in Mozambican children reported no clear-cut association between SMA and NTS bacteremia, although few children had NTS bacteremia $(n=12) \cdot{ }^{13}$ In agreement with the current study, previous studies have found no association between malaria and risk of NTS bacteremia, ${ }^{4,23}$ although other studies have reported mixed findings with malaria both reducing and increasing risk of NTS bacteremia. ${ }^{29-32}$ These differences might be explained by the prevalence of malarial anemia within the study populations or various other factors, including nutritional status. Taken to- 
gether, our findings suggest that SMA, rather than other malarial phenotypes, underlies the association between malaria and NTS bacteremia. Indeed, severe anemia due to all causes was strongly associated with NTS bacteremia, even after excluding children with malaria parasitemia, in agreement with a previous study in Malawian children. ${ }^{33}$ A number of pathways may contribute to increased risk of NTS bacteremia in children with SMA including hemolysis, iron overload and upregulation of heme oxygenase-1 (Figure 1). ${ }^{14}$ Hepcidin may also influence risk of NTS bacteremia in SMA by controlling the availability of iron for bacterial growth. ${ }^{18-20}$ We observed that hepcidin levels and hepcidin/ferritin ratios were lower in hospitalized children with SMA compared to those with CM. In agreement, a study in Kenyan children found lower hepcidin levels in malaria patients with severe anemia compared to those with higher hemoglobin levels. ${ }^{34}$ In contrast, a study in Nigerian children found no difference in hepcidin levels between children with SMA and CM and higher hepcidin levels in uncomplicated compared to severe malaria. ${ }^{35}$ Our findings may be explained by the low hepcidin/ferritin ratio and higher STfR levels in SMA compared to CM, indicating increased erythropoietic activity. Severe anemia negatively regulates hepcidin production through the action of erythroferrone ${ }^{36}$ even in the presence of inflammation/infection $^{37,38}$ or sickle cell disease. ${ }^{39}$ Inflammation, as measured by ferritin and CRP, did not differ between the SMA and CM groups, although parasite density, known to correlate with hepcidin levels, ${ }^{40}$ was higher in $\mathrm{CM}$. In agreement with previous studies, ${ }^{41,42}$ we found higher hepcidin levels in children with severe malaria compared to those with asymptomatic parasitemia. This is likely to be due to increased inflammation in severe malaria, rather than the older age of the community-based children, since older children would be expected to have higher hepcidin levels than younger children. ${ }^{26,43}$

Iron is an essential nutrient for bacterial growth and ex vivo studies suggest that increased serum iron levels may stimulate the growth of various bacteria including Salmonella Typhimurium. ${ }^{44,45}$ In mouse models, reduced hepcidin levels are associated with increased susceptibility to NTS infections,,$^{18}$ although little is known about the role of hepcidin during NTS and malaria infections in children. In the current study, children with SMA+NTS had lower hepcidin levels and hepcidin/ferritin ratios than those with NTS alone; although STfR, CRP and ferritin levels did not differ between these groups. High hepcidin levels would be expected in children with NTS bacteremia since hepcidin is known to increase in response to inflammation and infection. A challenge infection study with Salmonella enterica Typhi in the United Kingdom identified higher hepcidin concentrations during acute infection. ${ }^{46}$ In vitro and murine studies also show that Salmonella Typhimurium may directly or indirectly upregulate hepcidin ex- pression and perturb iron regulatory pathways. ${ }^{20}$ Hepcidin concentrations may alter iron availability within the Salmonella-containing vacuole (SCV). Recent evidence indicates that hepcidin leads to increased degradation of ferroportin on the SCV, and limits the movement of iron into the SCV. ${ }^{21}$ However, this conflicts with an earlier report that ferroportin transports iron out of the $\mathrm{SCV}^{47}$ and these contradictions may be based on differences in experimental systems used..$^{48}$ It also remains controversial whether iron accumulation in the SCV may promote bacterial growth by increasing iron availability, ${ }^{19,22}$ or kill bacteria through the Fenton reaction..$^{21}$ Low hepcidin levels in mice with severe hemolytic anemia were associated with increased susceptibility to Salmonella Typhimurium infection and hepcidin treatment improved survival. ${ }^{18}$ We hypothesize that low hepcidin levels in children with SMA, and non-malaria severe anemia, might contribute to NTS bacteremia by increasing iron availability in the SCV for NTS growth together with other mechanisms (Figure 1). Surprisingly, sTfR levels were elevated in children with NTS alone despite higher hemoglobin levels. It is not known whether NTS might induce transcription of transferrin receptors to increase transferrin iron acquisition, since transferrin receptors have been observed on the SCV during early phases of NTS endocytosis in murine models. ${ }^{49}$ To the best of our knowledge, this is the first study reporting hepcidin levels in children with NTS or with concomitant SMA and NTS bacteremia. The strengths of the study are that we utilized a very large 21 -year dataset $(n=75,034)$ with matching stored samples to identify and describe associations between severe malaria, NTS bacteremia and hepcidin in children. Our study also has important limitations. First, the study was observational, and as such, associations may be subject to unmeasured confounders and reverse causality. Second, we had few samples for children with SMA and NTS co-infection and no samples for those with $\mathrm{CM}$ and NTS co-infection since some samples were insufficient or missing, which may have introduced selection bias. Nonetheless, these are a unique sample set with accompanying clinical data collected over 21 years. Another limitation is that we did not measure additional parameters such as serum iron, transferrin saturation, and haptoglobin levels due to the volumes and availability of stored samples. Additionally, a few participants had STfR concentrations above the cut-off values making it challenging to interpret findings from regression models for sTfR (Online Supplementary Table S3). Finally, our study was conducted in a single site. It is also possible that our study underestimated associations, considering the low sensitivity of blood cultures used to identify NTS. Nonetheless, this study complements the existing in vitro and animal data on the relationship between SMA and NTS bacteremia and provides preliminary evidence on the possible role of hepcidin in mediating this association. 
In conclusion, SMA was associated with a strongly increased risk of NTS bacteremia in children and reduced hepcidin levels were observed in children with SMA and SMA+NTS. The question of whether ferroportin transports iron into or out of the SCV remains an active area of research, ${ }^{21,47}$ and future findings may support our hypothesis or generate new ideas on how low hepcidin might mediate NTS susceptibility in children with SMA. Further studies are needed to understand the role of the hepcidin-ferroportin axis in susceptibility to NTS in human subjects, how hepcidin and iron disturbances might mediate susceptibility to bacteremia due to NTS or other organisms, and how $P$. falciparum, iron deficiency or other etiologies of severe anemia influence this relationship.

\section{Disclosures}

No conflicts of interest to disclose.

\section{Contributions}

SHA conceptualized and designed the study; SHA, MN, JAGS, and TNW supervised the study; SMU, JM, SMWarumba and AWM performed laboratory analyses; EM serotyped non-typhoidal Salmonella isolates; KMA, JMM, and SHA analyzed and interpreted the data; KMA and SHA wrote the original draft of the manuscript; KMA, JMM, SMU, $K M, J M, R M, A W M, M S$, SMohammed, EM, SMwarumba, NM, $P B, J A G S, M N, T N W$, and SHA revised subsequent drafts and approved the final draft for publication.

\section{Acknowledgments}

The authors would like to thank the children who participated in this study and their parents/guardians. This manuscript was submitted for publication with the permission of the Director of the Kenya Medical Research Institute (KEMRI).

\section{Funding}

This study was funded by Wellcome (grant numbers 110255 to SHA, 212600 to KMA, 202800 to TNW, and a core award to the KEMRI-Wellcome Trust Research Program [203077]). KMA, RMM, EM and JMM were supported by the DELTAS Africa Initiative [DEL-15-003]. The DELTAS Africa Initiative is an independent funding scheme of the African Academy of Sciences (AAS)'s Alliance for Accelerating Excellence in Science in Africa (AESA) and supported by the New Partnership for Africa's Development Planning and Coordinating Agency (NEPAD Agency) with funding from Wellcome [107769] and the UK government. The funders had no role in study design, data collection and analysis, decision to publish, or preparation of the manuscript.

\section{Data-sharing statement}

The data and analyses underlying this article are available in Harvard Dataverse at https://doi.org/ 10.7910/DVN/KXZWN6 and applications for data access can be made through the Kilifi Data Governance Committee cgmrc@kemri-wellcome.org.

\section{References}

1. World Health Organisation. World malaria report 2020: 20 years of global progress and challenges. 2020 [cited 14 ${ }^{\text {th }}$ May 2021]; Available from:

https://www.who.int/publications/i/item/9789240015791

2. G.B.D. 2017 Non-Typhoidal Salmonella Invasive Disease Collaborators. The global burden of non-typhoidal salmonella invasive disease: a systematic analysis for the Global Burden of Disease Study 2017. Lancet Infect Dis. 2019;19(12):1312-1324.

3. Feasey NA, Dougan G, Kingsley RA, Heyderman RS, Gordon MA. Invasive non-typhoidal salmonella disease: an emerging and neglected tropical disease in Africa. Lancet. 2012;379(9835):2489-2499.

4. Park SE, Pak GD, Aaby P, et al. The relationship between invasive nontyphoidal Salmonella disease, other bacterial bloodstream infections, and malaria in Sub-Saharan Africa. Clin Infect Dis. 2016; 62(Suppl 1):S23-31.

5. Biggs HM, Lester R, Nadjm B, et al. Invasive Salmonella infections in areas of high and low malaria transmission intensity in Tanzania. Clin Infect Dis. 2014; 58(5):638-647.

6. Tabu C, Breiman RF, Ochieng B, et al. Differing burden and epidemiology of non-Typhi Salmonella bacteremia in rural and urban Kenya, 2006-2009. PLoS One. 2012;7(2):e31237.

7. Scott JA, Berkley JA, Mwangi I, et al. Relation between falciparum malaria and bacteraemia in Kenyan children: a population-based, case-control study and a longitudinal study. Lancet. 2011;378(9799):1316-1323.
8. Mackenzie G, Ceesay SJ, Hill PC, et al. A decline in the incidence of invasive non-typhoidal Salmonella infection in The Gambia temporally associated with a decline in malaria infection. PLoS One. 2010;5(5):e10568.

9. Bronzan RN, Taylor TE, Mwenechanya J, et al. Bacteremia in Malawian children with severe malaria: prevalence, etiology, HIV coinfection, and outcome. J Infect Dis. 2007;195(6):895-904.

10. Graham SM, Mwenechanya J, Tembo M, et al. The pattern of bacteraemia in children with severe malaria. Malawi Med J. 2002;14(1):11-15.

11. Nadjm B, Amos B, Mtove G, et al. WHO guidelines for antimicrobial treatment in children admitted to hospital in an area of intense Plasmodium falciparum transmission: prospective study. BMJ. 2010;340:c1350.

12. Falay D, Kuijpers LM, Phoba MF, et al. Microbiological, clinical and molecular findings of non-typhoidal Salmonella bloodstream infections associated with malaria, Oriental Province, Democratic Republic of the Congo. BMC Infect Dis. 2016;16:271

13. Bassat Q, Guinovart C, Sigaúque B, et al. Severe malaria and concomitant bacteraemia in children admitted to a rural Mozambican hospital. Trop Med Int Health. 2009;14(9):1011-1019.

14. Abuga KM, Muriuki JM, Williams TN, Atkinson SH. How severe anaemia might influence the risk of invasive bacterial infections in African children. Int J Mol Sci. 2020;21(18):6976.

15. Cunnington AJ, de Souza JB, Walther M, Riley EM. Malaria 
impairs resistance to Salmonella through heme- and heme oxygenase-dependent dysfunctional granulocyte mobilization. Nat Med. 2011;18(1):120-127.

16. Lokken KL, Stull-Lane AR, Poels K, Tsolis RM. Malaria parasitemediated alteration of macrophage function and increased iron availability predispose to disseminated nontyphoidal Salmonella infection. Infect Immun. 2018;86(9):e00301-00318.

17. Pagani A, Nai A, Silvestri L, Camaschella C. Hepcidin and anemia: a tight relationship. Front Physiol. 2019;10:1294.

18. Yuki KE, Eva MM, Richer E, et al. Suppression of hepcidin expression and iron overload mediate Salmonella susceptibility in ankyrin $1 \mathrm{ENU}$-induced mutant. PLoS One. 2013;8(2):e55331.

19. Liu D, Gan ZS, Ma W, et al. Synthetic porcine Hepcidin exhibits different roles in Escherichia coli and Salmonella infections. Antimicrob Agents Chemother. 2017;61(10):e02638-16.

20. Kim DK, Jeong JH, Lee JM, et al. Inverse agonist of estrogenrelated receptor gamma controls Salmonella typhimurium infection by modulating host iron homeostasis. Nat Med. 2014;20(4):419-424.

21. Lim D, Kim KS, Jeong JH, et al. The hepcidin-ferroportin axis controls the iron content of Salmonella-containing vacuoles in macrophages. Nat Commun. 2018;9(1):2091.

22. Flannagan RS, Farrell TJ, Trothen SM, Dikeakos JD, Heinrichs DE. Rapid removal of phagosomal ferroportin in macrophages contributes to nutritional immunity. Blood Adv. 2021;5(2):459-474.

23. Muthumbi E, Morpeth SC, Ooko M, et al. Invasive Salmonellosis in Kilifi, Kenya. Clin Infect Dis. 2015;61(Suppl 4):S290-301.

24. Bejon P, Williams TN, Liljander A, et al. Stable and unstable malaria hotspots in longitudinal cohort studies in Kenya. PLoS Med. 2010;7(7):e1000304.

25. Berkley JA, Lowe BS, Mwangi I, et al. Bacteremia among children admitted to a rural hospital in Kenya. N Engl $J$ Med. 2005;352(1):39-47.

26. Atkinson $\mathrm{SH}$, Uyoga $\mathrm{SM}$, Armitage $\mathrm{AE}$, et al. Malaria and age variably but critically control Hepcidin throughout childhood in Kenya. EBioMedicine. 2015;2(10):1478-1486.

27. World Health Organisation. Severe malaria. 2014 [cited November 17, 2020]; Available from: https://www.who.int/malaria/publications/atoz/who-severemalaria-tmih-supplement-2014.pdf

28. Enwere G, Van Hensbroek MB, Adegbola R, et al. Bacteraemia in cerebral malaria. Ann Trop Paediatr. 1998;18(4):275-278.

29. Brent AJ, Oundo JO, Mwangi I, Ochola L, Lowe B, Berkley JA. Salmonella bacteremia in Kenyan children. Pediatr Infect Dis J. 2006;25(3):230-236.

30. Mandomando I, Bassat Q, Sigauque B, et al. Invasive Salmonella infections among children from rural Mozambique, 2001-2014. Clin Infect Dis. 2015;61(Suppl 4):S339-345.

31. Mabey DC, Brown A, Greenwood BM. Plasmodium falciparum malaria and Salmonella infections in Gambian children. J Infect Dis. 1987;155(6):1319-1321.

32. Walsh AL, Phiri AJ, Graham SM, Molyneux EM, Molyneux ME. Bacteremia in febrile Malawian children: clinical and microbiologic features. Pediatr Infect Dis J. 2000;19(4):312-318.

33. Calis JC, Phiri KS, Faragher EB, et al. Severe anemia in Malawian children. N Engl J Med. 2008;358(9):888-899.

34. Casals-Pascual C, Huang H, Lakhal-Littleton S, et al. Hepcidin demonstrates a biphasic association with anemia in acute Plasmodium falciparum malaria. Haematologica. 2012;97(11):1695-1698.

35. Burte F, Brown BJ, Orimadegun AE, et al. Circulatory hepcidin is associated with the anti-inflammatory response but not with iron or anemic status in childhood malaria. Blood. 2013;121(15):3016-3022.

36. Latour C, Wlodarczyk MF, Jung G, et al. Erythroferrone contributes to hepcidin repression in a mouse model of malarial anemia. Haematologica. 2017;102(1):60-68.

37. Jonker FA, Calis JC, Phiri K, et al. Low hepcidin levels in severely anemic malawian children with high incidence of infectious diseases and bone marrow iron deficiency. PLoS One. 2013;8(12):e78964.

38. Stoffel NU, Lazrak M, Bellitir S, et al. The opposing effects of acute inflammation and iron deficiency anemia on serum hepcidin and iron absorption in young women. Haematologica. 2019;104(6):1143-1149.

39. Mangaonkar AA, Thawer F, Son J, et al. Regulation of iron homeostasis through the erythroferrone-hepcidin axis in sickle cell disease. Br J Haematol. 2020;189(6):1204-1209.

40. Howard CT, McKakpo US, Quakyi IA, et al. Relationship of hepcidin with parasitemia and anemia among patients with uncomplicated Plasmodium falciparum malaria in Ghana. Am J Trop Med Hyg. 2007;77(4):623-626.

41. Oluboyo OA, Theodora I, Oluboyo A. Impact of malaria severity on serum levels of hepcidin and iron status in children. Online $J$ Health Allied Sciences. 2019;18(1):1-4.

42. Mendonca VR, Souza LC, Garcia GC, et al. Associations between hepcidin and immune response in individuals with hyperbilirubinaemia and severe malaria due to Plasmodium vivax infection. Malar J. 2015;14(1):407.

43. Muriuki JM, Mentzer AJ, Webb EL, et al. Estimating the burden of iron deficiency among African children. BMC Med. 2020;18(1):31.

44. Prentice S, Jallow AT, Sinjanka E, et al. Hepcidin mediates hypoferremia and reduces the growth potential of bacteria in the immediate post-natal period in human neonates. Sci Rep. 2019;9(1):16596.

45. Cross JH, Bradbury RS, Fulford AJ, et al. Oral iron acutely elevates bacterial growth in human serum. Sci Rep. 2015;5:16670.

46. Darton TC, Blohmke CJ, Giannoulatou E, et al. Rapidly escalating hepcidin and associated serum iron starvation are features of the acute response to typhoid infection in humans. PLoS Negl Trop Dis. 2015;9(9):e0004029.

47. Chlosta S, Fishman DS, Harrington L, et al. The iron efflux protein ferroportin regulates the intracellular growth of Salmonella enterica. Infect Immun. 2006;74(5):3065-3067.

48. Nairz M, Weiss G. Iron in infection and immunity. Mol Aspects Med. 2020;75:100864.

49. Steele-Mortimer O, Meresse S, Gorvel JP, Toh BH, Finlay BB. Biogenesis of Salmonella typhimurium-containing vacuoles in epithelial cells involves interactions with the early endocytic pathway. Cell Microbiol. 1999;1(1):33-49.

50. World Health Organisation. WHO child growth standards based on length/height, weight and age. Acta Paediatr Suppl. 2006;450:76-85. 